\title{
Research on FeO Content of Sinter
}

\author{
Hu Qichen ${ }^{1}$, Wu Chunliang ${ }^{2}$, Sun Jianghuan ${ }^{1}$, Wang Na ${ }^{2, *}$, Hu Binsheng ${ }^{3}$ \\ ${ }^{1}$ Iron and Steel Research Institute of HBIS Group, Shijiazhuang, P. R. China \\ ${ }^{2}$ Research Institute for Hebei Vanadium Titanium Industry Technology, Chengde, P. R. China \\ ${ }^{3}$ School of Metallurgical and Energy, Hebei United University, Tangshan, P. R. China
}

Email address:

huqichen01@126.com (Hu Qichen), huchunliang@hbisco.com (Wu Chunliang),wnawjnw@126.com (Wang Na)

${ }^{*}$ Corresponding author

\section{To cite this article:}

$\mathrm{Hu}$ Qichen, Wu Chunliang, Sun Jianghuan, Wang Na, Hu Binsheng. Research on FeO Content of Sinter. International Journal of Mineral Processing and Extractive Metallurgy. Vol. 4, No. 1, 2019, pp. 1-6. doi: 10.11648/j.ijmpem.20190401.11

Received: December 11, 2018; Accepted: January 2, 2019; Published: February 15, 2019

\begin{abstract}
The FeO content of sinter is the key role for sinter quality, carbon consumption and production cost. And it has great effect on production status, fuel ratio and hot metal output of blast furnace. According to the relevant national standards and industry standards, the particle size, reducibility, drum strength, fuel ratio of sinter and BF production status in Tangsteel iron-making plant were analyzed in this paper. It is found that the optimal range of $\mathrm{FeO}$ content in Tangsteel is from 8.0 to $8.5 w t . \%$.
\end{abstract}

Keywords: Sinter, FeO, Drum Strength, Fuel Ratio

\section{Introduction}

The FeO content of sinter is very important for efficient production. It directly affects the particle size and cold strength of BF raw materials, thus affecting the reduction performance of $\mathrm{FeO}$. In the process of iron-making, the sinter with high $\mathrm{FeO}$ content has poor reducibility, which will affect the sTablele operation of blast furnace and not meet the need of smelting process. Therefore, the study on sinter with suiTablele $\mathrm{FeO}$ content has attracted much attention. In foreign advanced iron and steel enterprises (such as Japan and France), the reasonable range of $\mathrm{FeO}$ content of sinter is 4.0-5.0 wt.\% [1]. In domestic enterprises (such as Baosteel, Wusteel, Jisteel, Ansteel), the $\mathrm{FeO}$ content of sinter is controlled at about $7.0 \mathrm{wt} . \%$ [2-5], while for many small and medium-sized steel enterprises in China, the $\mathrm{FeO}$ content of sinter is relatively high, even more $10.0 \mathrm{wt} \%$ [6-8]. The control of $\mathrm{FeO}$ content of sinter has been a controversial issue in Tangsteel iron-making plant. For example, the $\mathrm{FeO}$ content of sinter in Tangsteel iron-making plant had fluctuated within the range of 12.0-9.16 wt.\% since the end of 2007 , which resulted in many production problems, such as the large fluctuation of sinter particle size, unsTablele blast condition, wind pressure fluctuation, and especially the great fluctuation of static pressure, unsTablele wall body, difficult control of furnace temperature and heat, daily coke ratio fluctuation around $10 \mathrm{Kg} / \mathrm{t} \mathrm{Fe}$. The $\mathrm{FeO}$ content of sinter was controlled between 8.0-9.0 wt.\% after 2009, which brought the sTablele furnace condition, degressive operation difficulty, and easy control for BF production [9-11]. Therefore, FeO content of sinter must be studied in blast furnace production. The effect of $\mathrm{FeO}$ content in sinter on raw material granularity, reduction performance, drum strength and blast furnace fuel ratio are researched in this paper.

\section{Experiment}

\subsection{Sample Preparation}

According to the literature review, if the $\mathrm{FeO}$ content of sinter is in the range of $7.0-9.0 \mathrm{wt} . \%$, the blast furnace production will be sTablele, the operation will be easy and $\mathrm{BF}$ production will be easy to control. Therefore, the experimental test points are randomly distributed within the range of 7.0-9.0 wt.\% FeO content so as to find the sTablele region.

\subsection{Experimental Procedures}

\subsubsection{Particle Size Test with Different FeO Content}

The raw material of experiment is the sinter, used in blast 
furnace in Tangsteel iron making plant. After screening, the size composition range of sinter was $<5 \mathrm{~mm}, 5-10 \mathrm{~mm}, 10-16$ $\mathrm{mm}, 16-25 \mathrm{~mm}, 25-40 \mathrm{~mm}$ and $>40 \mathrm{~mm}$.

\subsubsection{Reducibility Test with Different FeO Content}

Based on Iron ores-determination of reducibility $(\mathrm{GB} /$ t13241-91), the reduction test of sinter, used in Tangsteel iron-making plant, was studied in this paper.

The conditions were as follows: the reaction tube with inner diameter of $75 \mathrm{~mm}$; the sample mass was $500 \mathrm{~g}$; the particle size was $10.0-12.5 \mathrm{~mm}$; the ratio of reduced gas composition $\mathrm{CO}$ to $\mathrm{N}_{2}$ was $3 / 7$, the reduced gas flow rate was $15 \mathrm{~L} / \mathrm{min}$; the reduction temperature was $900 \mathrm{C}$ and the reaction time was $180 \mathrm{~min}$. The reducibility of sinter was measured as medium temperature reduction index (RI).

\subsubsection{Reducibility Test with Different FeO Content}

According to iron ores-low temperature disintegration testmethod using cold tumbling after static reduction (GB/ T13242-91), the low temperature disintegration of sinter was researched in this paper.

The low temperature degradation test was composed of constant temperature reduction test and drum test. The conditions of former test were that the inner diameter of reaction tube was $75 \mathrm{~mm}$, the sample mass was $500 \mathrm{~g}$, the particle size was $10.0-12.5 \mathrm{~mm}$, the reduction gas was composed of $\mathrm{CO}_{2}-30 \mathrm{CO}-60 \mathrm{~N}_{2}$, the reduction temperature was $500 \mathrm{C}$, the gas flow rate was $151 / \mathrm{min}$, and the reduction time was $60 \mathrm{~min}$. After the reduction reaction, it was cooled to room temperature in $\mathrm{N}_{2}$ atmosphere, and then weighted and loaded into a drum with $\varphi 130 \times 200 \mathrm{~mm}$ to do the drum test. The rotational speed was $30 \mathrm{rpm}$ and total round number was 300 . Then it was respectively sieved by $6.3 \mathrm{~mm}, 3.15 \mathrm{~mm}$ and $0.5 \mathrm{~mm}$ square hole screen to get corresponding particle size. And the mass percent of samples larger than $6.3 \mathrm{~mm}$, less than $3.15 \mathrm{~mm}$ and less than $0.5 \mathrm{~mm}$ were respectively presented as $\mathrm{RDI}_{6.3}, \mathrm{RDI}_{-3.15}$ and $\mathrm{RDI}_{-0.5}$, which were taken as the indicators of low temperature reduction performance of sinter.

\subsubsection{Reducibility Test with Different FeO Content}

The experiment adopted the 1/2ISO standard drum with diameter of $1000 \times 250 \mathrm{~mm}$. The sinter with particle size greater than $10 \mathrm{~mm}$ was $7.5 \mathrm{~kg}$, which was rotated at $25 \mathrm{rpm}$ for $8 \mathrm{~min}$. The mass percent of particle size greater than 6.3 $\mathrm{mm}$ was taken as the drum index, and the mass percent of particle size less than $0.5 \mathrm{~mm}$ as abrasion index.

\section{Results and Discussion}

\subsection{Effect on Sinter Property}

\subsubsection{Effect on Sinter Granularity}

Table 1. Particle size data of sinter with different FeO content.

\begin{tabular}{|c|c|c|c|c|c|c|}
\hline $\mathrm{FeO} / w t . \%$ & $<5 \mathrm{~mm}$ & $5-10 \mathrm{~mm}$ & $10-16 \mathrm{~mm}$ & $16-25 \mathrm{~mm}$ & $25-40 \mathrm{~mm}$ & $>40 \mathrm{~mm}$ \\
\hline 7.5 & 2.3 & 18.8 & 25.9 & 25.6 & 19.7 & 7.8 \\
\hline 8.0 & 2.3 & 18.0 & 26.4 & 26.8 & 18.5 & 7.9 \\
\hline 8.5 & 2.2 & 18.3 & 26.1 & 25.5 & 20.1 & 7.9 \\
\hline 9.0 & 2.2 & 18.1 & 25.5 & 26.4 & 20.1 & 7.8 \\
\hline
\end{tabular}

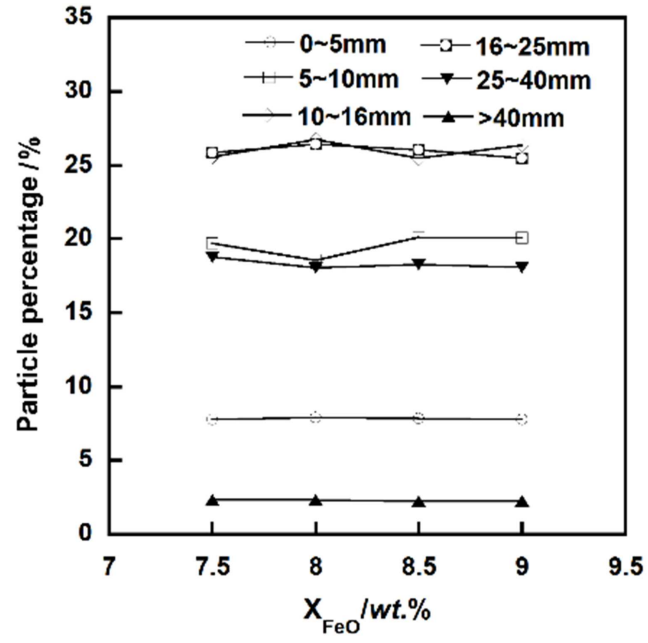

Figure 1. Distribution curve of particle size of sinter with different $\mathrm{FeO}$ content.

The particle size test result of sinter adopted in Tangsteel iron-making plant is shown in Tablele1 and Figure 1.
According to the experimental data and curve trend of Tablele1 and Figure 1, it can be seen that the particle size of sinter in the range of less than $5 \mathrm{~mm}$ decreases slightly with the increasing of $\mathrm{FeO}$ content from $7.5 w t . \%$ to $8.5 w t . \%$, and then increases slightly with the increasing of $\mathrm{FeO}$ content from $8.5 \mathrm{wt} . \%$ to $9.0 \mathrm{wt} . \%$, but the increasing quantity is not obvious. In the range of $5-10 \mathrm{~mm}$, the particle size of sinter has a waving trend with the increasing of $\mathrm{FeO}$ content, but the variation is not obvious. In the range of $10-16 \mathrm{~mm}$, the particle size of sinter is increased firstly and then decreases with the increasing of $\mathrm{FeO}$ content, and the maximum turning point is around $8.0 \mathrm{wt} . \%$, and the variation range is slightly larger than the previous two intervals. With the increasing of $\mathrm{FeO}$ content in the range of $16-25 \mathrm{~mm}$, the particle size variation is similar to that in the range of $5-10 \mathrm{~mm}$, but the fluctuation amplitude is relatively large, and the maximum value is $8.5 w t . \%$. With the increasing of $\mathrm{FeO}$ content in the range of $25-40 \mathrm{~mm}$, the particle size decreases firstly and then increases, and the lowest turning point is around 8.0 $w t . \%$. The variation range is larger than that in the range of $10-16 \mathrm{~mm}$. With the increasing of $\mathrm{FeO}$ content in the range of 
over $40 \mathrm{~mm}$, the particle size increases first and then decreases. The maximum turning point is around $8.0 \mathrm{wt} . \%$ with a slight fluctuation. Based on the above experimental data and analysis results, it can be seen that the best comprehensive distribution area of sinter particle size is within the $\mathrm{FeO}$ content of 8.0-8.5wt.\%.

\subsubsection{Effect on Sinter Reducibility}

As shown in Figure 2, the experimental results show that the reduction of sinter decreased with the increasing of $\mathrm{FeO}$ content, and there is a maximum value of 83.76 in the range of $\mathrm{FeO}$ content $7.0-7.5 w t . \%$, while there is also a lowest value 81.61 in the range of $\mathrm{FeO}$ content $8.5-9.0$ $w t . \%$. The difference between the highest point and the lowest point is about 2.15, thus, the difference is not big. According to the above experimental results and analysis, $\mathrm{FeO}$ content has no significant influence on the reduction of sinter within the range of 7.0-9.0 wt.\%, but the overall trend is decreasing.

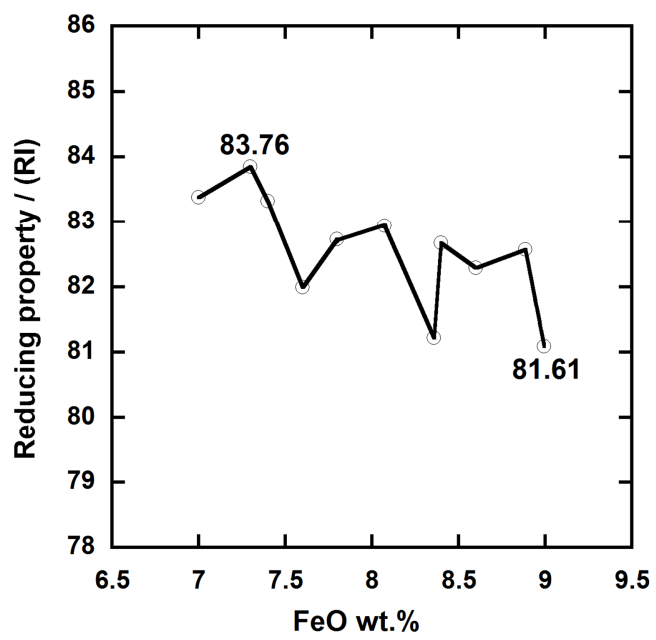

Figure 2. Curve of reduction variation of sinter with different $\mathrm{FeO}$ content.

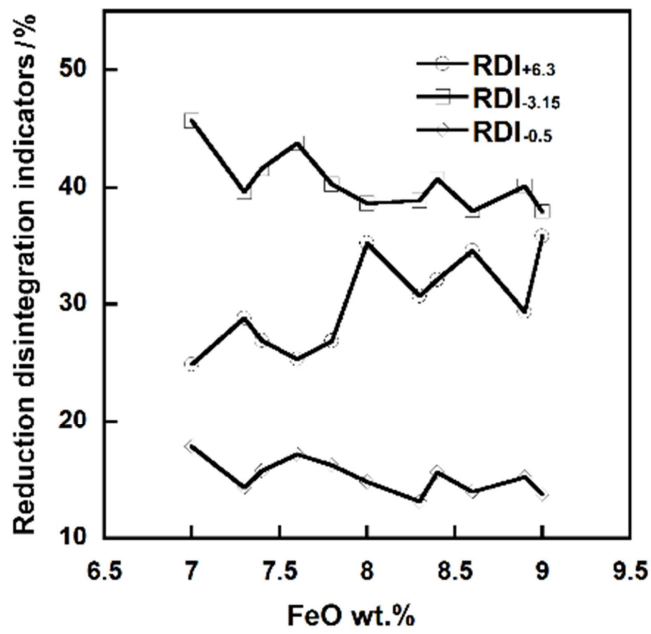

Figure 3. Curve of low-temperature reduction disintegration index with different $\mathrm{FeO}$ content.

Based on the $\mathrm{RDI}_{+6.3}$ curve of Figure 3, it can be seen that the overall trend of low temperature disintegration index is gradually decreased with the increasing of $\mathrm{FeO}$ content of sinter from $7.0 \mathrm{wt} \%$ to $9.0 \mathrm{wt} \%$, and when the $\mathrm{FeO}$ content is in the range of 8.0-9.0 wt.\%, the fluctuation extent of index is such small that it could be considered as no change; the overall trend of $\mathrm{RDI}_{-3.15}$ is gradually increased with the increasing of $\mathrm{FeO}$ content, and when the $\mathrm{FeO}$ content is in the range of 8.0-8.5 wt.\%, the increasing extent of index is very small, which can be considered as no change; the overall trend of $\mathrm{RDI}_{-0.5}$ is gradually decreased with the increasing of $\mathrm{FeO}$ content of sinter, but the decreasing extent is smaller than that of $\mathrm{RDI}_{+6.3}$, and when the $\mathrm{FeO}$ content is in the range of $8.0-9.0 w t . \%$, the increasing extent of index is very small, which can be considered as no change. From the experimental results and analysis, it can be seen that the excellent area of low temperature disintegration index of sinter is within the range of $8.0 \sim 8.5 w t . \% \mathrm{FeO}$ content.

\subsubsection{Effect on Sinter Tumbler Strength}

The influence of different $\mathrm{FeO}$ content on drum strength of sinter is shown in Figure.4. The drum strength of sinter increases with the increasing of $\mathrm{FeO}$ content in the range of $7.5-9.0 w t . \%$, but the increasing speed in the range of 7.5$8.0 w t . \%$ is slower than that in the range of 8.0-9.0 wt.\%, which could be seen from the slope change of curve segmentation. Therefore, according to the curve and analysis results of Figure 4, the $\mathrm{FeO}$ content of sinter has great influence on the drum strength. The overall trend of drum strength of sinter is generally increased within the range of 7.5-9.0 wt.\%.

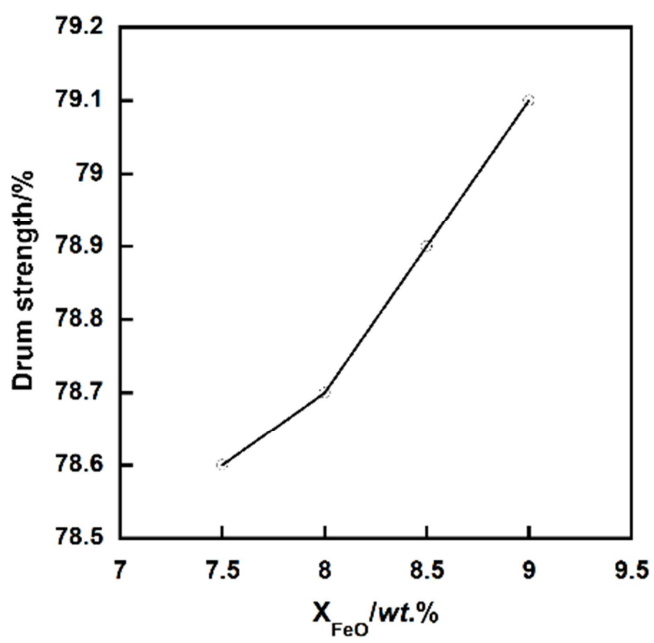

Figure 4. Curve of drum strength variation of sinter with different $\mathrm{FeO}$ content.

\subsubsection{Effect on Sinter Fuel Rate}

The variation of fuel ratio of sinter with different $\mathrm{FeO}$ content is shown in Figure 5. The FeO content has little influence on fuel ratio of sinter and the overall trend is gradually increased. In the range of $7.0-9.0 w t . \% \mathrm{FeO}$ content, the increasing extent is not significant. It is remarkable that the abnormal point (fuel ratio $629 \mathrm{~kg} / \mathrm{t}$ ) with about $8.5 w t . \%$ $\mathrm{FeO}$ content is a test point caused by the abnormal furnace 
body during the experimental process, which is regarded as an invalid point and can be ignored. Therefore, according to the above experimental results and analysis, it is considered that the fuel ratio of sinter is increased slightly with the increasing of $\mathrm{FeO}$ content in the range of 7.0-9.0 wt.\%, and its value fluctuation is within the normal index range of $\mathrm{BF}$ production.

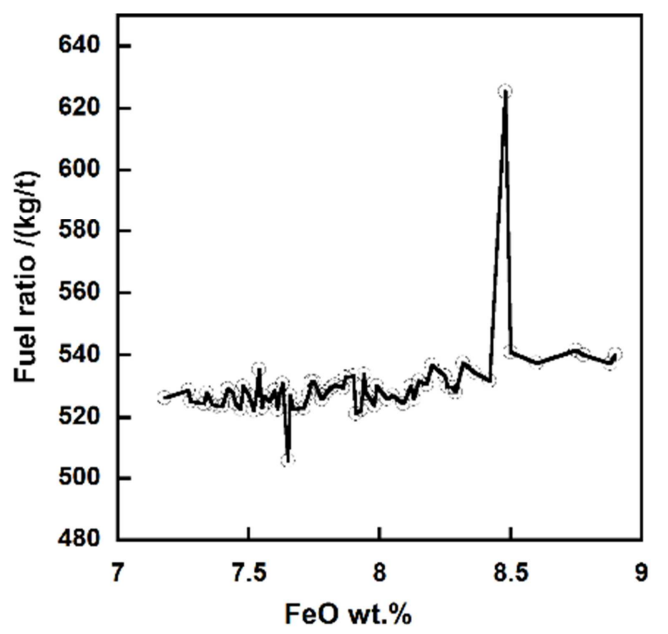

Figure 5. Curve of fuel ratio variation of sinter with different $\mathrm{FeO}$ content.

The influence of different $\mathrm{FeO}$ content on the utilization coefficient and gas utilization rate of blast furnace is shown in Figure 6. The $\mathrm{FeO}$ content of sinter has no significant influence on utilization coefficient and gas utilization rate of blast furnace. BF utilization coefficient is increased with the increasing of $\mathrm{FeO}$ content within the range of 7.0-9.0 wt.\%, while the increasing extent is slight and can be ignored. Therefore, according to the above experimental results and analysis, it is considered that $\mathrm{FeO}$ content within the range of $7.0-9.0 w t . \%$ has little influence on the utilization coefficient and gas utilization rate of blast furnace.

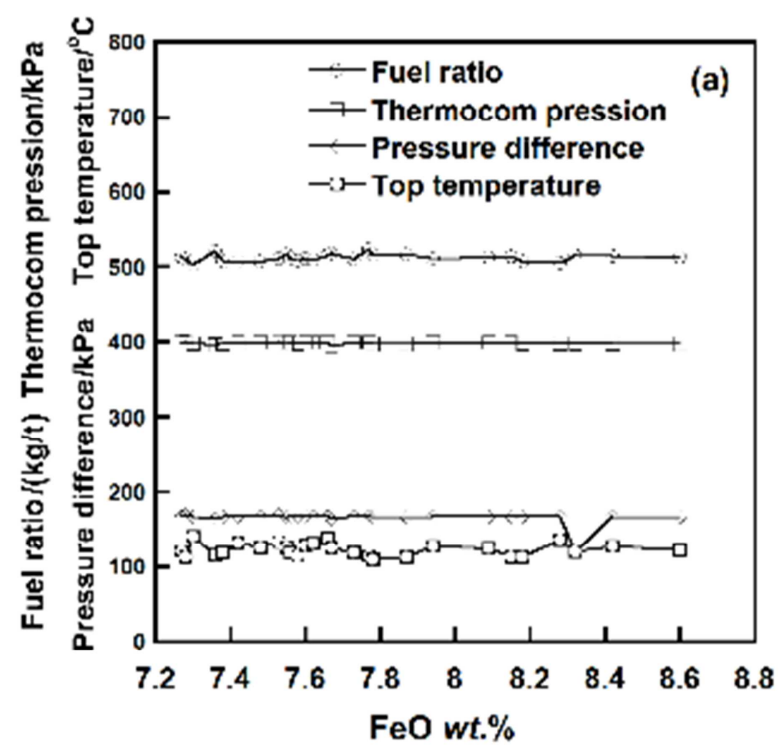

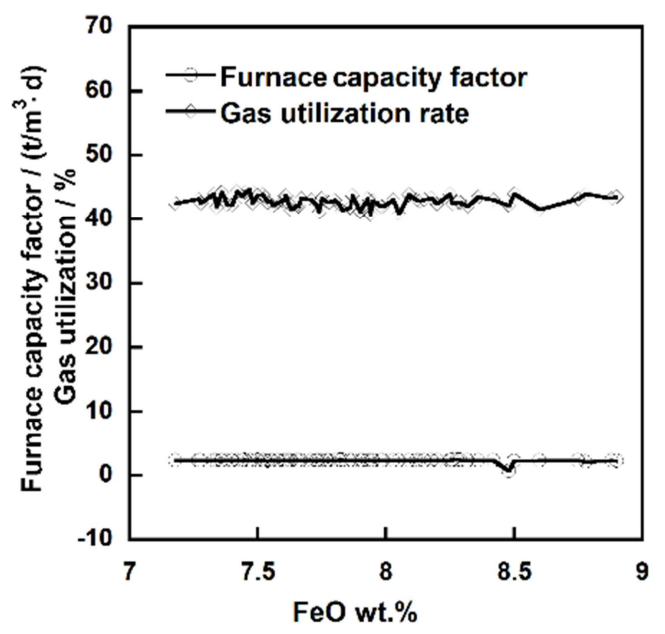

Figure 6. Curves of blast furnace utilization coefficient and gas utilization rate with different $\mathrm{FeO}$ content.

To sum up, according to the data analysis and research about the influence of different $\mathrm{FeO}$ content sinter on the particle size, reducibility, drum strength and fuel ratio, when the $\mathrm{FeO}$ content is within the range of 8.0-8.5 wt.\%, the sinter metallurgical properties are sTablele and conducive to the smooth production of blast furnace.

\subsection{Effect on Blast Furnace Production}

According to the previous study on the influence of different $\mathrm{FeO}$ content $(\mathrm{FeO}$ content within the range of 7.0$9.0 w t \%$ ) on particle size, reduction performance, drum strength and fuel ratio of sinter, it is found that the comprehensive performance index of sinter is better when the $\mathrm{FeO}$ content is within the range of 8.0-8.5 wt.\%. However, in order to verify whether the effect of different $\mathrm{FeO}$ content of sinter on blast furnace production is consistent with the experimental results, the actual production data of No.3 blast furnace in Tangsteel is tracked and collected, and the results are shown in Figure 7.

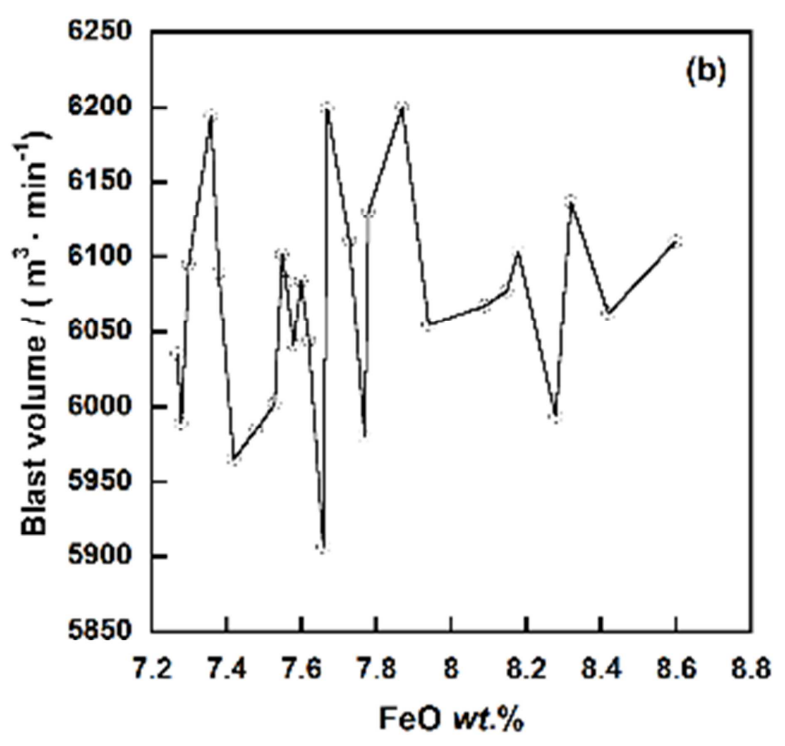



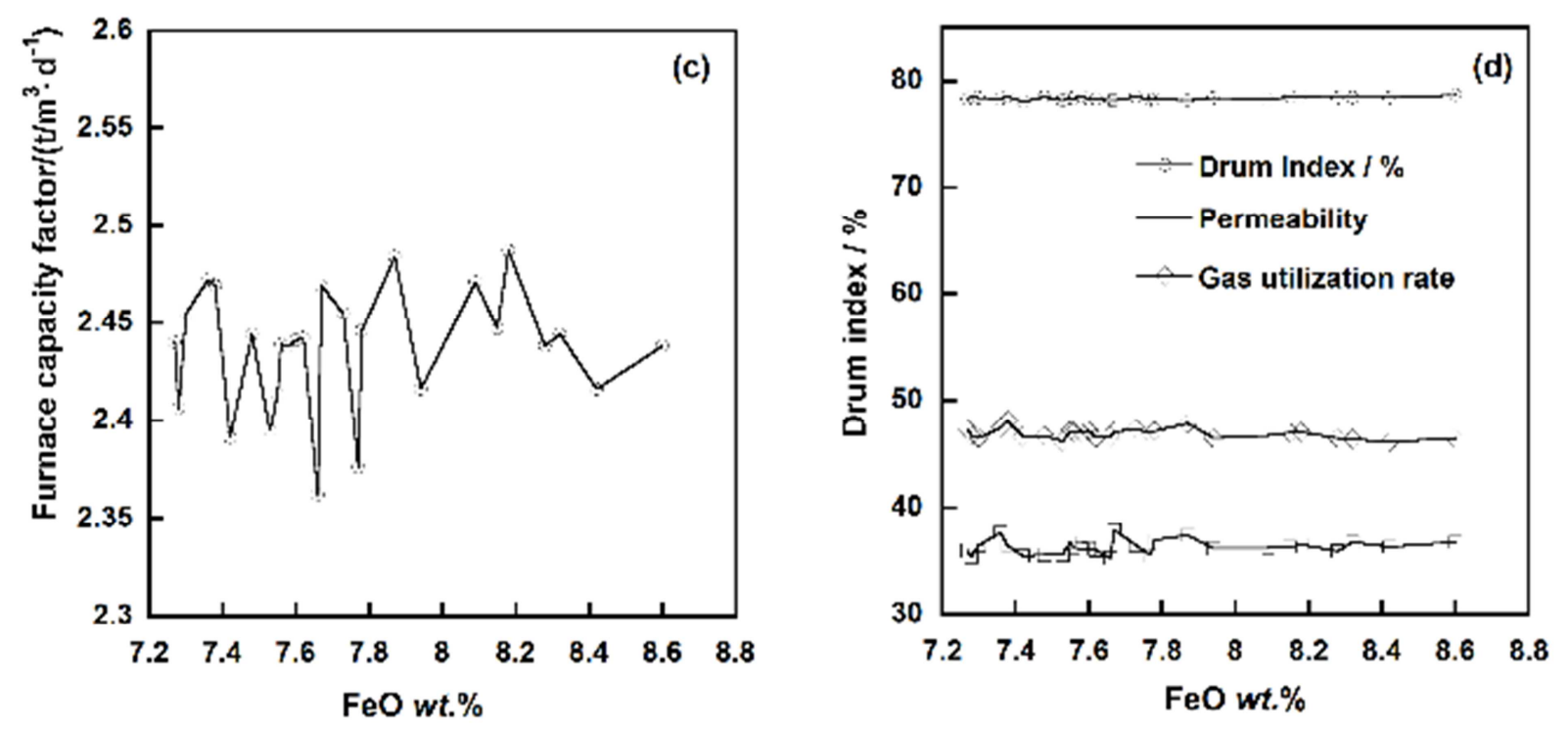

Figure 7. Curves of blast furnace production status with different $\mathrm{FeO}$ content of sinter: (a) fuel ratio, thermocompression, pressure difference and top temperature; (b) blast volume; (c) utilization coefficient of blast furnace; (d) drum index, permeability and gas utilization rate.

The curves about effect of different $\mathrm{FeO}$ content on fuel ratio, thermocompression, pressure difference and top temperature during the production of the blast furnace are shown in Figure 7(a). From the curves trend, it can be seen that the fluctuation of four factors gradually become sTablele with the increasing of $\mathrm{FeO}$ content of sinter, and the optimum $\mathrm{FeO}$ content range is $7.8-8.6 w t . \%$. It is noted that the large fluctuation of pressure difference within the range of 8.2-8.4 wt.\% is due to the normal maintenance of blast furnace.

The curve about influence of different $\mathrm{FeO}$ content on blast volume is shown in Figure 7 (b). From the curve trend, it can be seen that the fluctuation of blast volume gradually becomes sTablele with the increasing of $\mathrm{FeO}$ content of sinter, and the minimum fluctuation area is within the range of 7.98.6 w. $\% \mathrm{FeO}$ content.

The curve about influence of different $\mathrm{FeO}$ content on $\mathrm{BF}$ utilization coefficient is shown in Figure 7(c). The fluctuation frequency of utilization coefficient of blast furnace decreases with the increasing of $\mathrm{FeO}$ content of sinter. And the fluctuation of $\mathrm{BF}$ utilization coefficient is relatively slow within the range of $7.8-8.6 w t . \%$.

The curves about influence of different $\mathrm{FeO}$ content on drum index, permeability and gas utilization rate are shown in Figure 7(d). From the curves trend, it can be seen that the drum index has no obvious change with the increasing of $\mathrm{FeO}$ content, thus, the $\mathrm{FeO}$ content has little effect on drum index. However, the effect on sinter permeability and gas utilization rate is gradually reduced, and the most influential region is within the range of 7.8-8.6 wt.\%.

According to the collected data and analysis results, it is concluded that the $\mathrm{FeO}$ content has good influence on BF production within the range of 7.9-8.6 wt.\%, which is basically consistent with the previous experimental results (thus, the most suiTablele $\mathrm{FeO}$ content of sinter is 8.0-8.5 $w t . \%)$. Therefore, the experimental result in this paper has guiding significance for BF actual production in Tangsteel iron-making plant.

\section{Conclusions}

Based on the sampling research of metallurgical properties of sinter in Tangsteel iron-making plant and systematical study on the effect of sinter $\mathrm{FeO}$ content on particle size, reduction performance, drum strength and $\mathrm{BF}$ fuel ratio, the analysis results are as follows: The increasing of $\mathrm{FeO}$ content of sinter has little impact on particle size of raw material. The reduction performance and reduction degradation index of sinter slowly decrease, while the drum strength and blast furnace fuel ratio slowly increase with the increasing of $\mathrm{FeO}$ content. The best metallurgical property is within range of $8.0-8.5 w t . \% \mathrm{FeO}$ content. According to the analysis results of actual production data of No.3 blast furnace in Tangsteel iron-making plant, the comprehensive metallurgical property of sinter is the best within the range of $7.9-8.6 \mathrm{wt} \% \mathrm{FeO}$ content, which is basically consistent with the test result.

\section{Acknowledgements}

This work was supported by the National key research and development plan (No. 2017YFC0210600).

\section{References}

[1] Seah M P, Hordros E. Grain Boundary Segregation [J]. Proy. Roy. Soc. 1973, (335): 191-212.

[2] Liu zhulin. Study on influencing factors of $\mathrm{FeO}$ content in sinter [J]. Journal of chongqing institute of technology, 2005, 7 (1): 8-13.

[3] $\mathrm{Mu}$ jiyao. Soft melting properties of high alkalinity sinter [J]. Sintered pellets, 1986, (6): 17-23. 
[4] Wang changqiu, wukeng, houenjian, etc. Study on sintering carbon distribution and mineral distribution of thick material layer in east burning plant of anshan iron \& steel [J]. Journal of steel research, 2010, (6) : 14-17.

[5] Nachtrab W T, Chou Y T. High Temperature Ductility Loss incarb-manganese and niobium-treated Steel [J]. Metall. Trans. A, 1986, 17A (11): 1995-2006.

[6] Yin DE, li qiumei. Research and production practice of influencing factors of sinter $\mathrm{FeO}[\mathrm{J}]$. Sinter pellets, 1999 (5) : 31-33.

[7] Dai shuping, zhoulei. Effect of $\mathrm{FeO}$ content on mineral composition and microstructure of sinter [J]. Metal world, 2011, (6): 32-34.
[8] Huang yijun. Relationship between sinter $\mathrm{FeO}$ content and sinter production and quality [J]. Sinter pellets, 1980, (1) : 1726.

[9] Cheng zhenxian. Several questions about $\mathrm{FeO}$ in the sinter [J]. Sintered pellets.1983, (3): 33-38.

[10] Shan jiguo. Study on low temperature reduction degradation of sinter [J]. Sintered pellets, 1989, (2) : 15-18.

[11] Zhang ling. Reduction of $\mathrm{FeO}$ content in sinter to improve metallurgical properties of sinter [J]. Jiangsu metallurgy, 1998, (3): $32-33$. 